\title{
Even-odd flux quanta effect in the Fraunhofer oscillations of an edge-channel Josephson junction
}

\author{
B. Baxevanis, V. P. Ostroukh, and C. W. J. Beenakker \\ Instituut-Lorentz, Universiteit Leiden, P.O. Box 9506, 2300 RA Leiden, The Netherlands
}

(Received 26 November 2014; published 30 January 2015)

\begin{abstract}
We calculate the beating of $h / 2 e$ and $h / e$ periodic oscillations of the flux-dependent critical supercurrent $I_{c}(\Phi)$ through a quantum spin-Hall insulator between two superconducting electrodes. A conducting pathway along the superconductor connects the helical edge channels via a nonhelical channel, allowing an electron incident on the superconductor along one edge to be Andreev reflected along the opposite edge. In the limit of small Andreev reflection probability the resulting even-odd effect is described by $I_{c} \propto \mid \cos (e \Phi / \hbar)+f$, with $|f| \ll 1$ proportional to the probability for phase-coherent interedge transmission. Because the sign of $f$ depends on microscopic details, a sample-dependent inversion of the alternation of large and small peaks is a distinctive feature of the beating mechanism for the even-odd effect.
\end{abstract}

DOI: 10.1103/PhysRevB.91.041409

PACS number(s): 73.23.-b, 74.45.+c, 74.78.Na

Superconductor-normal-metal-superconductor junctions with edge channel conduction in the normal region are governed by the interplay of charge $e$ and charge $2 e$ transport: Charge can only enter or exit the superconductor in units of $2 e$, but in the normal region this Cooper pair can be split over opposite edges, when an electron incident on the normalsuperconductor (NS) interface along one edge is Andreev reflected as a hole along the opposite edge.

For quantum Hall edge channels this mechanism produces Fraunhofer oscillations (oscillations of the critical current with enclosed flux $\Phi$ ) having a fundamental period of $h / e$, twice the usual periodicity [1]. These are chiral edge channels, so Andreev reflection along the edge of incidence is forbidden and only the circulating path of Fig. 1(a) contributes to the supercurrent. When the edge channels allow for propagation in both directions, the critical current includes the usual $h / 2 e$ periodic contributions from Andreev reflection along a single edge, and further $h / e$ periodic contributions from circulating paths without charge transfer [Fig. 1(b)].

Here we investigate this beating of $h / e$ and $h / 2 e$ periodic contributions to the Fraunhofer oscillations. We are motivated by recent work on proximity induced superconductivity in quantum spin-Hall (QSH) insulators [2-8], which in one series of experiments [4] showed Fraunhofer oscillations with an even-odd effect: Large peaks in the critical current at even multiples of $h / 2 e$ alternate with smaller peaks at odd multiples.

The QSH insulator has helical edge channels (with direction of motion tied to the spin), so we consider that case in what follows (although the beating mechanism for the even-odd effect does not rely on helicity). Following Ref. [6] we assume that the superconductors dope the contacted QSH insulator, locally pushing the Fermi level in the conduction band. The broad conducting pathway that appears along the NS interface will be gapped by the superconducting proximity effect, but a narrow gapless channel may remain because superconductivity only becomes effective at some penetration length $\xi_{0}$ from the NS interface. (Reference [4] estimates $\xi_{0} \gtrsim 240 \mathrm{~nm}$, comparable to the estimated width of the edge states.) This channel provides a connection between the helical edge states that is nonhelical, meaning that either spin can propagate in both directions.
To describe the phase-coherent coupling of helical and nonhelical edge channels we study a network model of the Josephson junction, inspired by the spectral theory of graphs [9] and as a counterpart to network models of the quantum Hall effect [10,11]. As we will show, all information on the temperature and flux dependence of the supercurrent can be encoded in the product of a permutation matrix, representing the connectivity of the network, and a block-diagonal matrix describing the relation between incoming and outgoing modes at each node of the network.

Edge-channel Josephson junction. We consider the Josephson junction geometry of Fig. 2(a). A current $I$ is passed between two superconducting electrodes at phase difference $\phi$, related to the voltage $V$ over the junction by the Josephson relation $d \phi / d t=(2 e / \hbar) V$. Upon increasing the current bias, the junction switches from zero to finite dc voltage at a critical current $I_{c}$, dependent on the enclosed magnetic flux $\Phi$. If phase fluctuations can be neglected (for a low-impedance environment), the critical current is given by

$$
I_{c}(\Phi)=\max _{\phi}|I(\phi, \Phi)|
$$

We seek the oscillatory $\Phi$ dependence of $I_{c}$ (Fraunhofer oscillations) in a junction where the current flows along the edges, rather than through the bulk.

Referring to Fig. 2(b), the junction has width $W$ (edges at $x=0, W)$ and length $L$ (NS interfaces at $y=0, L)$. We choose a gauge where the superconducting pair potential $\Delta_{0}$ is real. A vector potential $\boldsymbol{A}=A_{y} \hat{y}$ in the $y$ direction,

$$
A_{y}=\frac{\Phi x}{L W}+\frac{\Phi_{0} \phi}{2 \pi} \delta(y-L / 2), \quad \Phi_{0} \equiv \frac{h}{2 e},
$$

then accounts for the phase difference between the NS interfaces.

Network model. To capture the essence of the problem, while still allowing for analytical solution, we represent the scattering processes by a network [Fig. 2(c)]. At the nodes $n=1,2,3,4$ the helical edge channels along $x=0, W$ are coupled to a single-mode nonhelical channel along $y=$ $0, L$. Each node has a $4 \times 4$ electronic scattering matrix $s_{n}$, which relates incoming and outgoing wave amplitudes of 
(a)

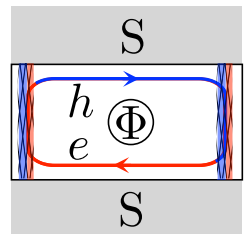

(b)

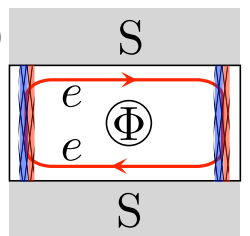

FIG. 1. (Color online) Beating mechanism for the even-odd effect in the Fraunhofer oscillations. For uncoupled edges the flux periodicity is $h / 2 e$, corresponding to the transfer of a charge- $2 e$ Cooper pair along the left or right edge channel [blue (red) hatched strips]. The edge channels are coupled by a conducting path along the NS interface, allowing for a circulating loop of charge $\pm e$ with $h / e$ flux periodicity. The circulating loop may be partly $e$ type (red lines) and partly $h$ type (blue), as in panel (a), or it may be entirely of one charge type [entirely $e$, as in panel (b), or entirely $h$ ]. Both loops contribute to the even-odd effect, but panel (a) dominates when the Andreev reflection probability $\Gamma$ is small. [It is of order $\Gamma$, while panel (b) is of order $\Gamma^{2}$.]

the helical channel, $a=\left(a_{\uparrow}, a_{\downarrow}\right)$, and the nonhelical channel, $b=\left(b_{\uparrow}, b_{\downarrow}\right)$, according to

$$
\left(\begin{array}{l}
a \\
b
\end{array}\right)_{\text {out }}=s_{n}\left(\begin{array}{l}
a \\
b
\end{array}\right)_{\text {in }} .
$$

The short-range scattering at a node can be taken as energy independent, so the hole scattering matrix is simply the complex conjugate $s_{n}^{*}$. We collect these matrices in the unitary matrix $s_{\text {node }}=s_{1} \oplus s_{1}^{*} \oplus \cdots \oplus s_{4} \oplus s_{4}^{*}$, consisting of eight $4 \times 4$ blocks arranged along the diagonal.

Since the effect of the magnetic field is only felt on long length scales, we can assume that $s_{n}$ preserves time-reversal symmetry. The requirement

$$
s_{n}=\left(\begin{array}{cc}
\sigma_{y} & 0 \\
0 & \sigma_{y}
\end{array}\right) s_{n}^{\mathrm{T}}\left(\begin{array}{cc}
\sigma_{y} & 0 \\
0 & \sigma_{y}
\end{array}\right),
$$

(a)

(c)
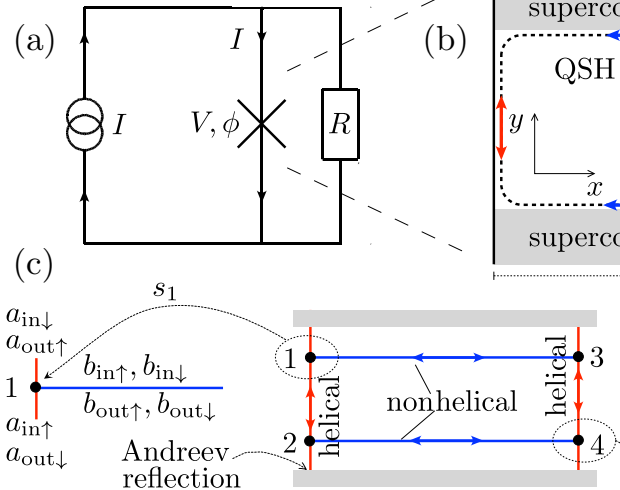

(b)

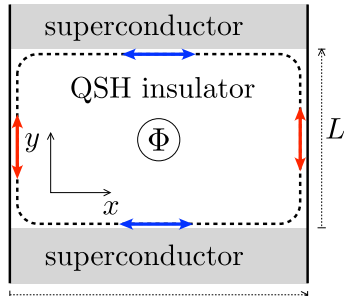

$W$

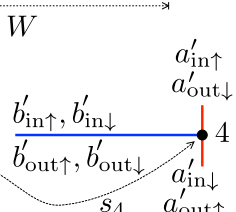

FIG. 2. (Color online) Josephson junction in a current-biased circuit [panel (a)], to study the dependence of the critical current $I_{c}$ on the magnetic flux $\Phi$ enclosed by a circulating edge channel [panel (b)]. The network model of the Josephson junction is illustrated in panel (c). Helical modes (red, amplitudes $a_{\uparrow}, a_{\downarrow}$ ) and nonhelical modes (blue, amplitudes $b_{\uparrow}, b_{\downarrow}$ ) are coupled at four nodes by a scattering matrix $s_{n}$, relating incoming and outgoing amplitudes.

together with unitarity, $s_{n}^{\dagger} s_{n}=1$, imposes the form [12]

$$
s_{n}=\left(\begin{array}{cc}
e^{2 i \psi_{n}} \sigma_{0} \sqrt{\Gamma_{n}} & e^{i \psi_{n}+i \psi_{n}^{\prime}} U_{n} \sqrt{1-\Gamma_{n}} \\
e^{i \psi_{n}+i \psi_{n}^{\prime}} U_{n}^{\dagger} \sqrt{1-\Gamma_{n}} & -e^{2 i \psi_{n}^{\prime}} \sigma_{0} \sqrt{\Gamma_{n}}
\end{array}\right) .
$$

Helical and nonhelical channels are coupled with probability $1-\Gamma_{n}$, while $U_{n} \in \mathrm{SU}(2)$ describes the spin-mixing associated with that coupling. [Equation (4) is satisfied because $\sigma_{y} U_{n}^{\mathrm{T}} \sigma_{y}=U_{n}^{\dagger}$ for any $\mathrm{SU}(2)$ matrix $U(n)$.] Time-reversal symmetry forbids spin mixing within the helical or nonhelical channel, which is why the upper-left and lower-right blocks of $s_{n}$ are proportional to the $2 \times 2$ unit matrix $\sigma_{0}$.

The nodes are connected by a unitary bond matrix $s_{\text {bond }}$, which is the product of a diagonal matrix of phase factors and a permutation matrix. We decompose $s_{\text {bond }}=s_{\text {left }} \oplus s_{\text {right }} \oplus$ $s_{\text {bottom }} \oplus s_{\text {top }}$ in terms of matrices $s_{\text {left }}$ and $s_{\text {right }}$ that connect the $a$ amplitudes (along $x=0$ and $x=W$, with phase factor $\left.e^{i \varepsilon L / \hbar v} \exp \left[i \tau_{z}(e / \hbar) \int A_{y} d y\right]\right)$ and matrices $s_{\text {bottom }}$ and $s_{\text {top }}$ that connect the $b$ amplitudes (along $y=0$ and $y=L$, with phase factor $\left.e^{i \varepsilon W / \hbar v}\right)$. Andreev reflection is included in $s_{\text {left }}$ and $s_{\text {right }}$ via matrix elements that connect a node to itself, switching electron-hole and spin band with phase factor

$$
s_{\mathrm{A}}=i \alpha \tau_{y} \otimes \sigma_{y}, \quad \alpha(\varepsilon)=i \varepsilon / \Delta_{0}+\sqrt{1-\varepsilon^{2} / \Delta_{0}^{2}} .
$$

(The Pauli matrices $\tau_{i}$ and $\sigma_{i}$ act, respectively, on the electronhole $e, h$ and spin $\uparrow, \downarrow$ degree of freedom.)

Knowledge of $s_{\text {node }}$ and $s_{\text {bond }}$ determines the entire spectrum of the network [9]. A bound state at energy $|\varepsilon|<\Delta_{0}$ corresponds to a unit eigenvalue of $M(\varepsilon)=s_{\text {node }} S_{\text {bond }}(\varepsilon)$, leading to the determinantal equation $\operatorname{det}[1-M(\varepsilon)]=0$. The density of states of the continuous spectrum at $|\varepsilon|>\Delta_{0}$ is given by [13]

$$
\rho(\varepsilon)=-\frac{1}{\pi} \frac{d}{d \varepsilon} \operatorname{Im} \ln \operatorname{det}\left[1-M\left(\varepsilon+i 0^{+}\right)\right]+\text {constant, }
$$

where the "constant" refers to $\phi$-independent terms. The Josephson current at temperature $T$ then follows from $[16,17]$

$$
I(\phi, \Phi)=-k T \frac{2 e}{\hbar} \frac{d}{d \phi} \sum_{p=0}^{\infty} \ln \operatorname{det}\left[1-M\left(i \omega_{p}\right)\right],
$$

as a sum over fermionic Matsubara frequencies $\omega_{p}=(2 p+$ 1) $\pi k T$. This expression assumes that the system equilibrates without restrictions on the fermion parity, so it holds on time scales long compared to the quasiparticle poisoning time (otherwise there would appear an additional sum over bosonic Matsubara frequencies) [18].

Uncoupled edges. When $k T \gg \hbar v / W$ there is no phasecoherent coupling between the edges at $x=0$ and $x=W$. We may then set $s_{\text {top }}$ and $s_{\text {bottom }}$ to zero in the evaluation of the determinant in Eq. (8), with the result

$$
I(\phi, \Phi)=I_{\text {edge }}(\phi)+I_{\text {edge }}\left(\phi+2 \pi \Phi / \Phi_{0}\right),
$$

$$
\begin{gathered}
I_{\text {edge }}(\phi)=k T \frac{4 e}{\hbar} \sin \phi \sum_{p=0}^{\infty}\left[2 \cos \phi+\zeta\left(\omega_{p}\right)+1 / \zeta\left(\omega_{p}\right)\right]^{-1}, \\
\zeta(\omega)=\Gamma^{2} e^{-2 \omega L / \hbar v}\left[\sqrt{1+\omega^{2} / \Delta_{0}^{2}}-\omega / \Delta_{0}\right]^{2} .
\end{gathered}
$$

(To simplify the formulas we have taken identical $\Gamma_{n} \equiv \Gamma$.) 
For $\Gamma \rightarrow 1$ we recover the short-junction-to-long-junction crossover formula of Ref. [18], which in the short-junction limit $L \ll \hbar v / \Delta_{0}$ and for low temperatures $k T \ll \Delta_{0}$ results in a critical current

$$
I_{c}(\Phi)=\frac{e \Delta_{0}}{2 \hbar}\left(1+\left|\cos \left(\pi \Phi / \Phi_{0}\right)\right|\right)
$$

with minima that are offset from zero, in agreement with Ref. [6]. For $\Gamma \ll 1$, still in the short-junction and lowtemperature limit, we find instead

$$
\begin{aligned}
& I(\phi, \Phi)=I_{0} \sin \left(\phi+\pi \Phi / \Phi_{0}\right) \cos \left(\pi \Phi / \Phi_{0}\right), \\
\Rightarrow & I_{c}(\Phi)=I_{0}\left|\cos \left(\pi \Phi / \Phi_{0}\right)\right|, \quad I_{0}=\frac{8 e \Delta_{0}}{3 \pi \hbar} \Gamma^{2} .
\end{aligned}
$$

For these uncoupled edges the critical current is $h / 2 e$ periodic in $\Phi$.

Coupled edges. The effect on the supercurrent of a phasecoherent coupling of the edges can be studied perturbatively in powers of $e^{-\pi k T W / \hbar v}$, by expanding the logarithmic determinant in Eq. (8) with the help of the formula

$$
\begin{aligned}
\ln \operatorname{det}\left(1-M_{0}-\delta M\right)= & \ln \operatorname{det}\left(1-M_{0}\right) \\
& -\sum_{n=1}^{\infty} \frac{1}{n} \operatorname{Tr}\left[\left(1-M_{0}\right)^{-1} \delta M\right]^{n} .
\end{aligned}
$$

The lowest order contribution with $h / e$ periodicity in $\Phi$ is given by

$$
\begin{aligned}
\delta I_{h / e}= & k T \frac{2 e}{\hbar} \frac{d}{d \phi} \operatorname{Tr} s_{\text {node }}\left(1-s_{\text {left }} s_{\text {node }}\right)^{-1} s_{\text {top }} s_{\text {node }} \\
& \left.\cdot\left(1-s_{\text {right }} s_{\text {node }}\right)^{-1} s_{\text {bottom }}\right|_{\varepsilon=i \omega_{0}}+\left\{s_{\text {left }} \leftrightarrow s_{\text {right }}\right\},
\end{aligned}
$$

describing a quasiparticle that encircles the junction clockwise or anticlockwise.

The effect of this contribution is largest for small Andreev reflection probability $\Gamma_{n} \ll 1$. To first order in $\Gamma$, and in the low-temperature, short-junction limit, we find

$$
\begin{aligned}
\delta I_{h / e}= & (8 e / \hbar) k T e^{-2 \pi k T W / \hbar v} \sin \left(\phi+\pi \Phi / \Phi_{0}\right) \\
& \times\left(\sqrt{\Gamma_{1} \Gamma_{2}}+\sqrt{\Gamma_{1} \Gamma_{4}}+\sqrt{\Gamma_{3} \Gamma_{4}}+\sqrt{\Gamma_{3} \Gamma_{2}}\right) \\
& \times \sin \left(\gamma_{2}-\gamma_{4}\right) \sin \left(\gamma_{1}-\gamma_{3}\right) .
\end{aligned}
$$

(To simplify a lengthy general expression we made a definite choice $U_{n}=e^{i \gamma_{n} \sigma_{x}}, \psi_{n}=\psi_{n}^{\prime}=0$ for the spin-mixing matrices.) Without spin mixing, for $\gamma_{n}=0$, the contribution (16) of order $\Gamma$ vanishes, but there is a nonzero contribution of order $\Gamma^{2}$,

$$
\begin{aligned}
\delta I_{h / e}= & (8 e / \hbar) k T e^{-2 \pi k T W / \hbar v}\left\{\left[\sin \left(\phi-\pi \Phi / \Phi_{0}\right) \Gamma_{1} \Gamma_{2}\right.\right. \\
& \left.\left.+\sin \left(\phi+3 \pi \Phi / \Phi_{0}\right) \Gamma_{3} \Gamma_{4}\right]\right\} .
\end{aligned}
$$

The contributions (16) and (17) correspond to the pathways shown in Figs. 1(a) and 1(b), respectively.

The addition of $\delta I_{h / e}$ to the zeroth-order supercurrent (12) (for identical $\Gamma_{n} \equiv \Gamma$ ) gives the critical current

$$
\begin{aligned}
I_{c}(\Phi) & =I_{0}\left|\cos \left(\pi \Phi / \Phi_{0}\right)+f\right|, \\
f & =\frac{12 \pi k T}{\Delta_{0} \Gamma} e^{-2 \pi k T W / \hbar v} \sin \left(\gamma_{2}-\gamma_{4}\right) \sin \left(\gamma_{1}-\gamma_{3}\right),
\end{aligned}
$$

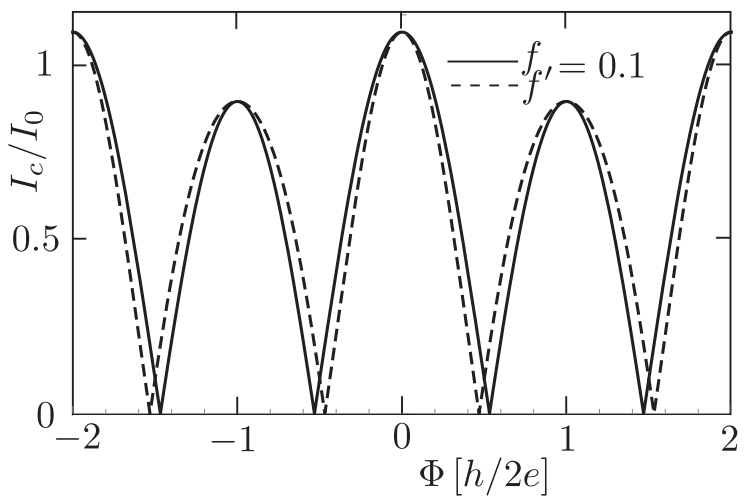

FIG. 3. Even-odd effect in the Fraunhofer oscillations of the critical current due to the beating of $h / e$ and $h / 2 e$ oscillations. The curves are calculated with spin mixing from Eq. (18) [solid lines, dominated by the path of Fig. 1(a)] and without spin mixing from Eq. (19) [dashed lines, dominated by the path of Fig. 1(b)].

with spin mixing at the nodes, and

$$
\begin{aligned}
I_{c}(\Phi) & =I_{0}\left|\cos \left(\pi \Phi / \Phi_{0}\right)+f^{\prime} \cos \left(2 \pi \Phi / \Phi_{0}\right)\right|, \\
f^{\prime} & =\left(6 \pi k T / \Delta_{0}\right) e^{-2 \pi k T W / \hbar v},
\end{aligned}
$$

without spin mixing. Both types of Fraunhofer oscillations are $h / e$ periodic, with an even-odd effect of relative magnitude $f$ or $f^{\prime}$ (see Fig. 3).

Comparison with experiment. Turning now to the experiment that motivated this analysis [4], we first of all notice that the observed even-odd effect appears already for the first few peaks around zero field. An explanation in terms of a Lorentz-force induced asymmetry in the current distribution is therefore unlikely [19-23]. The $h / e$-periodic Josephson effect of Majorana zero modes [24] is spoiled, on the time scale of the experiment, by any small amount of quasiparticle poisoning [6], so an explanation along these lines is not viable. A conducting pathway through the bulk, parallel to the edges, can explain the data [4] — but only if it is located within $10 \%$ of the device center (the flux $\Phi$ needs to be accurately partitioned into twice $\Phi / 2$ ). The mechanism proposed here does not require any such fine tuning.

The InAs/GaSb quantum well with Ti/Al electrodes of Ref. [4] has superconducting gap $\Delta_{0}=0.125 \mathrm{meV}$ and edge state velocity [25] $v=4.6 \times 10^{4} \mathrm{~m} / \mathrm{s}$. We take the same $v$ for the nonhelical channel. There is some uncertainty in the effective dimensions of the junction; we set $L=0.5 \mu \mathrm{m}, W=$ $3.5 \mu \mathrm{m}$. We then have comparable $L$ and $\xi_{0}=\hbar v / \Delta_{0}$, so we calculate the supercurrent directly from Eq. (8)—without taking the short-junction limit. The observed critical current in the $0.25 \mathrm{nA}$ range implies an Andreev reflection probability $\Gamma \approx 0.2$, which is the value we take for $\Gamma_{n}$ at all four scattering nodes.

The degree of spin mixing upon propagation along the nonhelical channel is quantified by setting $U_{1} U_{3}^{\dagger}=U_{2} U_{4}^{\dagger}=$ $e^{i \gamma \sigma_{x}}$. The value of $\gamma$ is unknown; we take a moderately strong spin mixing with $\gamma=\pi / 6$, but note that the even-odd effect exists also without any spin mixing (see Fig. 3). The critical current shown in Fig. 4 exhibits an even-odd effect of a similar 


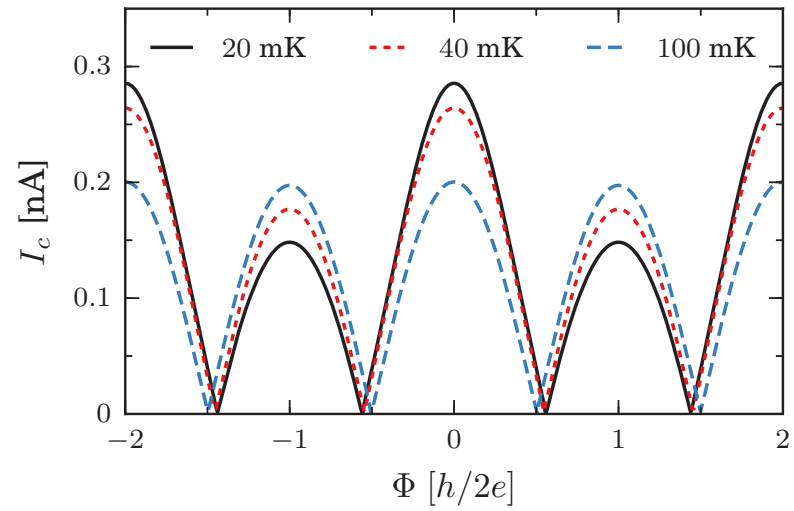

FIG. 4. (Color online) Fraunhofer oscillations for the experimentally relevant parameters given in the text, calculated from Eq. (8) for three different temperatures.

magnitude as observed experimentally [4]. The temperature dependence is somewhat stronger: In the experiment traces of the even-odd effect are still visible at $100 \mathrm{mK}$, but not in our calculation.

The beating mechanism has one qualitative feature that can help to distinguish it from other explanations of the even-odd effect: The sign of the effect-whether the $\Phi=0$ peak is larger or smaller than the $\Phi=h / 2 e$ peak-depends on microscopic details. This is evident from Eq. (18), in that the offset $f$ can be of either sign. A similar inversion of the even-odd effect can be induced by varying the phase shifts in the node scattering matrix (5), as we show in Fig. 5. Observation of an even-odd effect with the smallest peak at even multiples of $h / 2 e$ would constitute strong support for the beating mechanism, but no such inversion has been found so far [4].

In our analysis we have assumed helical edge state transport, appropriate for a quantum spin-Hall insulator, but the beating mechanism itself would apply also to nonhelical edge conduction. As was also pointed out in the experimental paper [4], the Fraunhofer oscillations are a sensitive probe of the current distribution, but cannot distinguish between a topologically trivial or nontrivial Josephson junction. That would require observation of a quantized conductance or supercurrent.

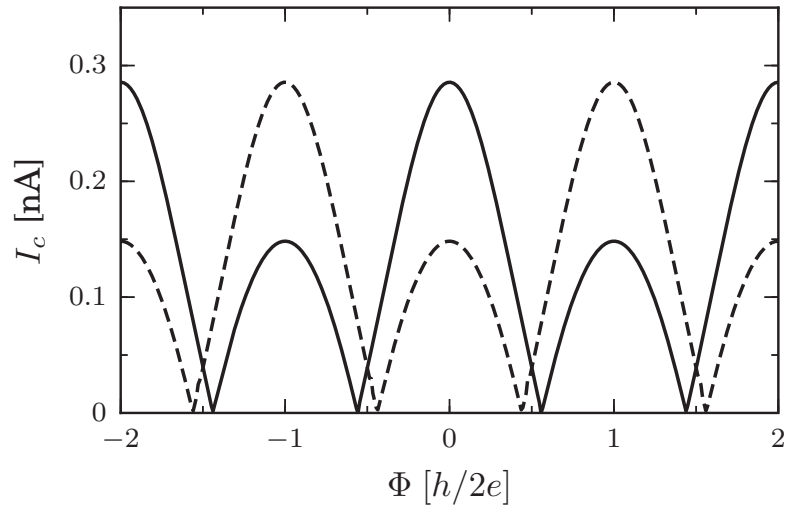

FIG. 5. The solid curve is the $T=20 \mathrm{mK}$ critical current of Fig. 4, without phase shifts at the scattering nodes, while the dashed curve shows the inverted even-odd effect for $\psi_{1}^{\prime}+\psi_{3}^{\prime}=\pi$ (and all other phase shifts kept at zero).

In conclusion, we have analyzed the effect of interedge coupling on the Fraunhofer oscillations in a quantum spin-Hall Josephson junction. A network model allows for an efficient description of the beating of $h / 2 e$ periodic intraedge and $h / e$ periodic interedge contributions to the critical current. The even-odd effect has comparable magnitude to what is observed in a recent experiment [4] (see Fig. 4), but the sample-dependent inversion of Fig. 5 has not been observed.

We note that the beating mechanism studied here in the two-dimensional geometry of a quantum spin-Hall insulator may apply more generally when a pair of conducting pathways enclosing different flux interferes. Indeed, a recent work studies a similar beating effect in a one-dimensional wire geometry [26], to explain multiperiodic Fraunhofer oscillations observed in Bi nanowires [27].

Acknowledgments. We acknowledge discussions on the experiment with L. P. Kouwenhoven and on the role of fermion-parity switches with I. C. Fulga, and we thank A. R. Akhmerov for a critical reading of the manuscript. This research was supported by the Foundation for Fundamental Research on Matter (FOM), the Netherlands Organization for Scientific Research (NWO/OCW), and an ERC Synergy Grant.
[1] J. A. M. van Ostaay, A. R. Akhmerov, and C. W. J. Beenakker, Phys. Rev. B 83, 195441 (2011).

[2] I. Knez, R.-R. Du, and G. Sullivan, Phys. Rev. Lett. 109, 186603 (2012).

[3] S. Hart, H. Ren, T. Wagner, P. Leubner, M. Mühlbauer, C. Brüne, H. Buhmann, L. W. Molenkamp, and A. Yacoby, Nat. Phys. 10, 638 (2014).

[4] V. S. Pribiag, A. J. A. Beukman, F. Qu, M. C. Cassidy, C. Charpentier, W. Wegscheider, and L. P. Kouwenhoven, Edge-mode superconductivity in a two dimensional topological insulator, arXiv:1408.1701.

[5] X. Shi, W. Yu, Z. Jiang, B. A. Bernevig, W. Pan, S. D. Hawkins, and J. F. Klem, Giant supercurrent states in a superconductor-InAs/GaSb-superconductor junction, arXiv: 1410.7342 .

[6] S.-P. Lee, K. Michaeli, J. Alicea, and A. Yacoby, Phys. Rev. Lett. 113, 197001 (2014).

[7] H.-Y. Hui, A. M. Lobos, J. D. Sau, and S. Das Sarma, Phys. Rev. B 90, 224517 (2014).

[8] G. Tkachov, P. Burset, B. Trauzettel, and E. M. Hankiewicz, Quantum interference of edge supercurrents in a twodimensional topological insulator, arXiv:1409.7301, calculate the flux dependence of $I_{m}(\Phi)=\left|I\left(\phi_{0}, \Phi\right)\right|$ at the fixed phase $\phi_{0}$ that maximizes the zero-field supercurrent $I(\phi, 0)$. This partial maximization provides a lower bound to the critical current $I_{c}(\Phi)=\max _{\phi}|I(\phi, \Phi)|$, but the flux periodicity of $I_{c}$ cannot be 
deduced from $I_{m}$. Although Tkachov et al. find an even-odd effect in $I_{m}$, the critical current has no even-odd effect in their model.

[9] U. Smilansky, J. Phys. A 42, 035101 (2009).

[10] J. T. Chalker and P. D. Coddington, J. Phys. C 21, 2665 (1988).

[11] B. Kramer, T. Ohtsuki, and S. Kettemann, Phys. Rep. 417, 211 (2005).

[12] Y. Jiang, X. Lu, and F. Zhai, Standard form of the scattering matrix for time reversal symmetric system, arXiv:1310.3733.

[13] See Supplemental Material at http://link.aps.org/supplemental/ 10.1103/PhysRevB.91.041409 for the derivation of Eq. (7), (see also, Refs. [14,15] and therein).

[14] D. Giuliano and I. Affleck, J. Stat. Mech. (2013) P02034.

[15] E. Akkermans, A. Auerbach, J. E. Avron, and B. Shapiro, Phys. Rev. Lett. 66, 76 (1991).

[16] C. W. J. Beenakker, Phys. Rev. Lett. 67, 3836 (1991); Three "universal" mesoscopic Josephson effects, extended version at arXiv:cond-mat/0406127.

[17] P. W. Brouwer and C. W. J. Beenakker, Chaos, Solitons \& Fractals 8, 1249 (1997); errata at arXiv:cond-mat/9611162.

[18] C. W. J. Beenakker, D. I. Pikulin, T. Hyart, H. Schomerus, and J. P. Dahlhaus, Phys. Rev. Lett. 110, 017003 (2013).
[19] In a device with aspect ratio $W / L$ the Lorentz force produces an even-odd effect in the Fraunhofer oscillations for flux $\Phi \gtrsim(W / L) h / e$, as calculated in Refs. [20,21] and measured in Refs. [22,23].

[20] U. Ledermann, A. L. Fauchère, and G. Blatter, Phys. Rev. B 59, R9027 (1999).

[21] V. Barzykin and A. M. Zagoskin, Superlattices Microstruct. 25, 797 (1999).

[22] J. P. Heida, B. J. van Wees, T. M. Klapwijk, and G. Borghs, Phys. Rev. B 57, R5618 (1998).

[23] Y. Harada, S. Jensen, T. Akazaki, and H. Takayanagi, Physica C 367, 229 (2002).

[24] L. Fu and C. L. Kane, Phys. Rev. B 79, 161408(R) (2009).

[25] Q. Z. Wang, X. Liu, H.-J. Zhang, N. Samarth, S.-C. Zhang, and C.-X. Liu, Phys. Rev. Lett. 113, 147201 (2014).

[26] S. V. Mironov, A. S. Mel'nikov, and A. I. Buzdin, Double path interference and magnetic oscillations in Cooper pair transport through a single nanowire, arXiv:1411.1626.

[27] C. Li, A. Kasumov, A. Murani, S. Sengupta, F. Fortuna, K. Napolskii, D. Koshkodaev, G. Tsirlina, Y. Kasumov, I. Khodos, R. Deblock, M. Ferrier, S. Guéron, and H. Bouchiat, Phys. Rev. B 90, 245427 (2014). 